\title{
Effect of Cerebrospinal Fluid on Fibroblasts Concerning Epidural Fibrosis: An In Vitro Study
}

\author{
Doğa Gürkanlar ${ }^{1}$, Sevda Lafc1 Fahrioğlü,4, Umut Fahrioğlu, ${ }^{3,4,5}$
}

\begin{abstract}
One of the most common treatments for lumbar disc herniation and other lumbar disorders is lumbar laminectomy. There may be some unwanted and serious complications with this procedure such as the "failed back surgery syndrome (FBSS)". Epidural fibrosis (EF), mainly due to fibroblast proliferation, emerges as the main cause of failed back surgery syndrome. According to the current literature and practice techniques, different agents are being used to prevent $\mathrm{EF}$ formation. To date there is no single agreed upon treatment method of EF. In this study, dilutional effect of CSF, together with low potassium levels, on primary skin fibroblast cultures was studied as a possible material for EF prevention. CSF at different concentrations (0-100\%) were tested to see its effect on Skin fibroblast proliferation. A wound healing assay was also performed to see the effect of CSF on wound healing. The cell proliferation goes up from $24 \mathrm{~h}$ to $72 \mathrm{hr}$ in all CSF percentages from $0-75 \%$ but the proliferation was inhibited at $100 \%$ CSF. The "wound" is closed successfully in all CSF percentages between 0-75. The $100 \%$ CSF fails to completely close the wound. Adverse effects of low concentrations of potassium levels and dilutional effect of CSF may be a promising solution in the prevention of EF. Further in vivo and in vitro experiments are required to characterize its use.
\end{abstract}

Keywords: Cerebrospinal Fluid; Epidural Fibrosis; Fibroblasts; in vitro

${ }^{1}$ Department of Neurosurgery, Faculty of Medicine, Near East University, Nicosia Cyprus ${ }^{2}$ Department of Anatomy, Faculty of Medicine, Near East University, Nicosia Cyprus

${ }^{3}$ Department of Medical Biology, Faculty of Medicine, Near East University, Nicosia Cyprus ${ }^{4}$ Near East University, Research Centre of Experimental Health Sciences, Nicosia, Cyprus ${ }^{5}$ Near East University, Genetics and Cancer Diagnosis-Treatment Centre, Centre of Excellence, Nicosia, Cyprus

${ }^{*}$ Corresponding author: Umut Fahrioğlu, $\mathrm{PhD}$ Department of Medical Biology, Faculty of Medicine, Near East University, Nicosia Cyprus Near East University, Research Centre of Experimental Health Sciences, Nicosia, Cyprus Near East University, Genetics and Cancer Diagnosis-Treatment Centre, Centre of

Excellence, Nicosia, Cyprus

Email: umut.fahrioglu@gmail.com or umutfahrioglu@gmail.com

DOI: $10.2478 /$ ebtj-2021-0019

(c) 2021 Authors. This work was licensed under the Creative Commons Attribution-NonCommercialNoDerivs 4.0 License.

\section{Introduction}

One of the most common treatments for lumbar disc herniation and other lumbar disorders is lumbar laminectomy. Unfortunately, there may be some unwanted and serious complications with this procedure such as the "failed back surgery syndrome (FBSS)" (1-3). FBSS is characterized by long term unsatisfactory relief or recurrence symptoms in patients who had laminectomies performed (4).

According to the literature, epidural fibrosis (EF), which emerges as the main cause of FBSS, results from the proliferation of fibroblasts, which is followed by the transformation of fibroblasts to myoblasts and the accumulation of disorganized ECM proteins (4-6). FBSS is seen in $8-48 \%$ of patients that have undergone lumbar disc surgery $(6,7)$. Postoperative epidural fibrosis is an unavoidable part of the healing process following lumbar disc surgery. As high as $25 \%$ of the FBSS case are thought to be due to EF. Lack of effective treatment against EF brings the attention back to surgical technique when it comes to scar formation (8). Epidural adhesions make re-exposure of the same operation area technically difficult and dangerous because of the greatly increased risk in nerve injury and dural tears (9).

According to the current literature and practice techniques, different agents are being used to prevent EF formation. Some of these agents are biological barriers such as animal collagen membranes, Gelofoamand there are also different varieties of biological and non-biological agents such as Adcon-L, honey and anti-inflammatory (7). To date there is no single agreed upon treatment method of EF (4). One strategy to reduce EF included the use of mitomycin $\mathrm{C}$, commonly used in cancer treatment, to induce apoptosis in the 
fibroblasts. Mitomycin achieves this by regulating the miR200b expression and thereby the RhoE expression (10).

Spinal surgery may also have other complications such as cerebrospinal fluid (CSF) fistula and pseudomeningocele, which may occur following an incidental durotomy (11-13).

In our clinical practice, we observed that the patients lack $\mathrm{EF}$ in the presence of CSF in epidural space. Therefore, we wanted to explore the effect of CSF on fibroblast activity in an attempt to prevent EF.

\section{Materials and Methods Cerebrospinal Fluid (CSF)}

Ethical approval was obtained from the University Scientific Research Ethics Evaluation Board (YDU/2017/44-372). CSF was obtained from patients with hydrocephalus, as part of a medically necessary procedure with the consent of the patient. The patients did not have cancer and were being followed for obstructive or communicating hydrocephalus. Following microbiological testing, CSF that has been deemed free of microorganisms has been selected for use in the experiment. CSF would be frozen right after collection at $-80^{\circ} \mathrm{C}$. Thawed and centrifuged (2000 rpm for 5 mins) to get rid of particulates and cells right before the experiment. CSF from three different patients were used for the experiments. Each CSF was used separately without mixing with other CSFs.

\section{Cell Culture}

Primary Human Dermal Fibroblasts (ATCC PCS-201-012) were used in this study. They were cultured using Serum Free-Fibroblast Growth Kit (ATCC 201-040) in Fibroblast Basal Medium (ATCC 201-030) according to the supplier's instructions. $0 \%, 25 \%, 50 \%, 75 \%$ and $100 \%$ CSF were used up to 72 hours. The $0 \%$ CFS contained no CSF and the $100 \%$ meant that the growth medium was completely replaced by the CSF. 25,50 and $75 \%$ CSF represent percentage of the total volume of the growth medium for the cells. The CSF used was human CSF and was not pooled together.

\section{Standard curves}

The standard curves are performed to find the range where the cell number will have a linear relationship with the relative Luminescence units (RLU). The CellTiter-Glo ${ }^{\circledR}$ (CTG) Luminescent Cell Viability Assay was used as per the manufacturer's instructions. Serial dilutions of ATCC PCS-201-012 cells were made $(5000,2500,1250,625$ and 312) in quadruplicate in a 96 well plate using growth medium. The CellTiter-Glo ${ }^{\oplus}$ Buffer was thawed and allowed to acclimate to room temperature. Room temperature lyophilized CellTiter-Glo ${ }^{\star}$ Substrate is then combined with the CellTiter-Glo ${ }^{\oplus}$ Buffer and vortexed to mix. $100 \mu \mathrm{l}$ of this picture is used for every well. Control wells without cells were also prepared to be used as a reference background read. Incubate for 2 minutes on an orbital shaker followed by a reading 1 hour after the addition of the mixture. The readings were taken with SpectraMax M5 Series by molecular devices using the SoftMax Pro software. Pre-set values for the CellTiter-Glo ${ }^{\circ}$
(CTG) Luminescent Cell Viability Assay were present in the software and is recommended for use. As the experiments will be performed in CSF a standard curve with different CSF percentages $(0 \%, 25 \%, 50 \%, 75 \%$ and $100 \%)$ was also performed.

\section{CellTiter-Glo • Luminescent Cell Viability Assay}

The CellTiter-Glo ${ }^{\circledast}$ Luminescent Cell Viability Assay was used as per the manufacturer's instructions. 3000 cells would be used in each well as standard starting cell count. Each experiment was done in quadruplicate (each specific CSF\% was seeded 4 different times for both the controls and cell containing experimental samples) and standard deviation was calculated. $0 \%, 25 \%, 50 \%, 75 \%$ and $100 \%$ CSF were tested on cells for 24 hours, 48 hours and 72 hours. 3 separate CSF's from 3 different individuals were tested. Controls with no cells were also made with $0 \%, 25 \%, 50 \%, 75 \%$ and $100 \%$ CSF. 396 well plates were used one for each of the days ( $24 \mathrm{~h}, 48 \mathrm{~h}$ and $72 \mathrm{~h})$.

\section{Wound Healing Assay}

6 well plates were seeded with cells and each well is allowed to reach $80 \%$ confluency. A straight-line scratch was made using a $200 \mu$ pipet tip in each well. Cells were washed with growth medium to get rid of debris. Confirmed that the scratch edges were smooth in the beginning of the experiment. Each scratch was incubated with different percentage of CSF $(0 \%, 25 \%, 50 \%$, $75 \%$ and $100 \%)$. Images of the cell proliferation activity were taken at 0 hours, 24 hours, 48 hours, 72 hours and 96 hours and $168 \mathrm{~h}$. The essay was performed in triplicate.

\section{Statistical analysis}

Descriptive statistics were calculated, and data were represented as arithmetic mean \pm standard error of mean (SEM) throughout the article. Standard curves for Cell number vs RLU and CFS vs RLU were drawn and linear regression analysis was performed to determine the equation of these two linear associations. In order to evaluate the effects of time and concentration, Two Way Repeated Measures Analysis of Variance (Two-Way RM ANOVA) model was used. In case of statistical significance, Holm-Sidak's multiple comparisons test was applied as post hoc analysis. Level of significance was accepted to be 0.05. All statistical calculations were carried out with GraphPad Prism software (Demo Version 8.2.1 for Mac).

\section{Results}

\section{Standard curves}

Serial dilutions of the ATCC PCS-201-012 cells were made from 5000 cells all the way down to 312 cells and each experiment was performed in quadruplicate. As mentioned earlier in the methods section, 3000 cells per well were used on a 96 well plate in the experiment which was in the linear range. The CTG system was also tested without cells with just the different percentage of CSFs used in the experiment. As the percentage of CSF goes up so does the RLU.

\section{CellTiter-Glo ${ }^{\bullet}$ Luminescent Cell Viability Assay}


Following the treatment of ATCC PCS-201-012 cells with various percentages of CSF, the cell viability, that is the number of cells present in the well, was determined using the CTG assay. $24 \mathrm{~h}, 48 \mathrm{~h}$ and 72 -hour results were obtained separately for each of the CSFs used. All CSFs followed a similar pattern of cell proliferation but CSF3 showed the most marked difference leading to a much greater cell proliferation even in the first 24 hours when compared to CSF1 and SCF2 (Figure 1). Figure 1 displays all three of the CSFs separately to display slightly dif- ferent growth response given to each CSF.

In order to simplify the data, the average of the all the CSF findings were also displayed separately in Figure 2 comparing cells viability only by hours and CSF percentages. Variation in cells number is reflected as a variance in luminescence and the two would be linearly correlated as indicated by the standard curves (data not shown). The cell proliferation goes up from $24 \mathrm{~h}$ to $72 \mathrm{hr}$ in all CSF percentages from $0-75 \%$ but the proliferation was inhibited at $100 \%$ CSF.

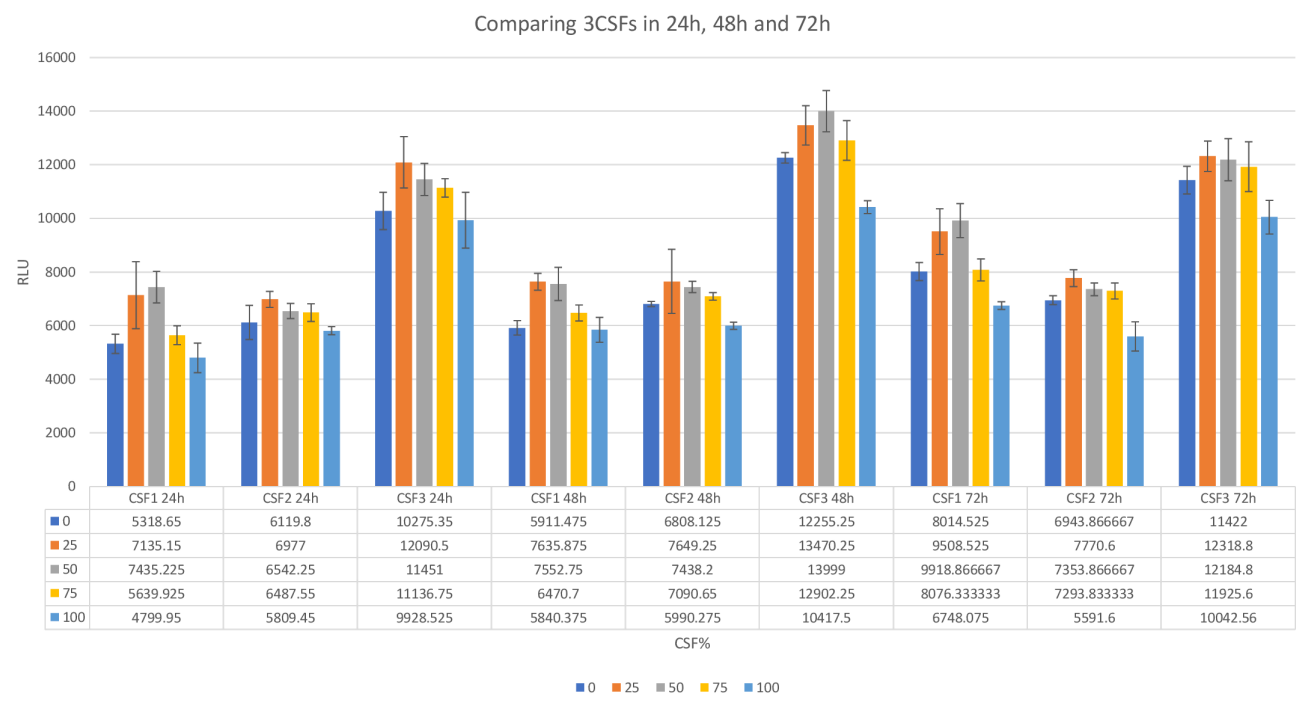

Figure 1. CTG Assay results using PCS-201-012 cells at 24h,48h and $72 \mathrm{~h}$ with $0 \%, 25 \%, 50 \%, 75 \%$ and $100 \%$ CSF. Three different CSFs were used in the experiments. All experiments were performed in quadruplicate. CSF 3 caused the highest amount of growth out of all the CSFs across all hours and all CSF percentages. There is inhibition of growth with the $75 \%$ and $100 \%$ but it is more prominent with $100 \%$ CSF. This can be seen in all of the time series. Two Way RM ANOVA with Holm-Sidak multiple comparison test was used for statistical analysis.

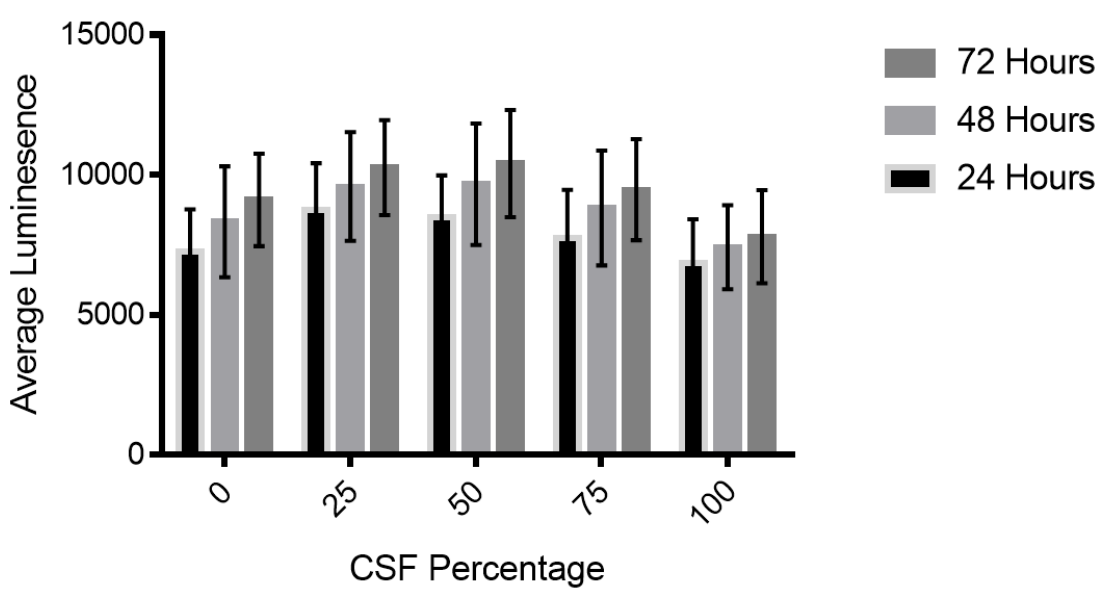

Figure 2. All the CSF results were combined and presented as one CSF. Variation in cell number can be seen as a variation in luminescence (RLU). The cell number goes up from $24 \mathrm{~h}$ to $72 \mathrm{~h}$ but in $100 \%$ CSF proliferation is inhibited. Two Way RM ANOVA with Holm-Sidak multiple comparison test was used for statistical analysis. 


\section{Statistical analysis}

Different variables such as time (in hours) and CSF values were analysed for their variance and also cross compared against each other with respect to their RLU values (and therefore cell number). The CSF percentage variability observed was observed to be statistically significant (Table 1).

Table 1. Relative Luminescence Unit (RLU) values are shown for different CSF concentrations and different hours. Same letters in the columns indicate no statistically significant difference $(p>0.05)$. Same symbols in the rows indicate no statistically significant difference $(p>0.05)$. The variability due to the CSF percentages was statistically significant. Two Way RM ANOVA with Holm-Sidak multiple comparison test was per-

\section{Discussion}

Epidural fibrosis (EF) was initially discussed in the literature in 1948. It is characterized by fibroblast accumulation, extracellular matrix protein deposits and the inflammation induced distortion of the normal tissue $(6,14)$. TGF-1b and IL-6, inflammatory cytokines, have been suggested to play a role in the development and promotion of $\mathrm{EF}(4)$. Fibroblasts produce large amounts of TGF-1b $(6,15)$.Excessive levels of TGF-1b stimulates fibroblast proliferation, and eventual differentiation of the myofibroblasts. This also accelerates the deposition of ECM by the myofibroblasts. The ECM proteins include fibronectin which will contribute to the process of fibrosis $(6,16)$. This cascade through TGF-1b is thought to be the main reason for ex-

Table 1. Relative Luminescence Unit (RLU) values are shown for different CSF concentrations and different hours. Same letters in the columns indicate no statistically significant difference ( $p>0.05)$. Same symbols in the rows indicate no statistically significant difference $(\mathrm{p}>0.05)$. The variability due to the CSF percentages was statistically significant. Two Way RM ANOVA with Holm-Sidak multiple comparison test was performed for this analysis.

\begin{tabular}{|c|c|c|c|c|}
\hline & & \multicolumn{3}{|c|}{ TIME } \\
\hline & & 24 Hours & 48 Hours & 72 Hours \\
\hline & & Mean \pm SEM & Mean \pm SEM & Mean \pm SEM \\
\hline \multirow{5}{*}{$\begin{array}{c}\text { CSF Concentration } \\
(\%)\end{array}$} & 0 & $7237.93 \pm 1536.22 \mathrm{a}, \mathrm{d}, \#$ & $8324.95 \pm 1982.12 \mathrm{a}, \&$ & $9103.60 \pm 1653.54 \mathrm{a},{ }^{*}$ \\
\hline & 25 & $8734.22 \pm 1678.76$ b,c, $\#$ & $9585.13 \pm 1942.57$ b,c, $\&$ & $10262.11 \pm 1698.34 \mathrm{~b}, \mathrm{c}, *$ \\
\hline & 50 & $8476.16 \pm 1509.59 c$ & $9663.32 \pm 2168.09 c, \&$ & $10404.24 \pm 1916.68 c,^{*}$ \\
\hline & 75 & $7754.74 \pm 1708.62 \mathrm{a}, \#$ & $8821.20 \pm 2048.36 \mathrm{a}, \&$ & $9473.12 \pm 1802.25 \mathrm{a}^{*}$ \\
\hline & 100 & $6845.98 \pm 1568.58 \mathrm{~d}, \#$ & $7416.05 \pm 1501.35 \mathrm{~d}, \#, \&$ & $7796.31 \pm 1660.38 \mathrm{~d}, \&$ \\
\hline
\end{tabular}

formed for this analysis.

For each given hour (24h, $48 \mathrm{~h}$ and $72 \mathrm{~h})$ different CSF percentages were compared to each other. Mean difference of each comparison and its statistical significance is indicated in Table 2.

Most of the comparisons were producing results of statistical significance. Table 3 shows the comparisons in the other way around where different time points were compared to each other within a specific CSF percentage. Most of these comparisons were also statistically significant.

\section{Wound Healing Assay}

The scratches formed were followed between 0 hours and 168 hours. Pictures taken every 24 hours with all the CSF percentages. Figure 4 shows representative pictures for specified percentages and hours. At 96 hours the wound was closed by almost all the CSF percentages except for the $100 \%$. The portions of $0 \%$ CSF show some unclosed patches, but the scar was mostly closed just like in the $25 \%$ CSF experiment. The unclosed patches were shown in Figure 3. The 100\% CSF fails to completely close the wound, but some fibroblasts can be seen extending into the "scar". All pictures were not displayed due to space constraints. 96 hours was chosen as it displays the most drastic contrast to the initial wound. and $1068.56 \mathrm{~cm}^{-1}$, ffectivel cessive epidural fibrosis in laminectomized spines $(5,6)$.

Many different methods and substances have been tried and experimented within an effort to prevent EF with varying degrees of success $(1,4,6-10,14,17-19)$. Some of these methods include the application of a physical barrier such as Adcon L and polytetrafluoroethylene membrane in an effort to prevent epidural adhesion. However, placement of these barriers and gel foams on the roots and dura post-surgery did not reach the desired effectiveness nor did they improve the magnetic resonance imaging (MRI) findings. The pharmaceutical agents and chemicals employed were not any better in preventing EF. They included many chemicals ranging from immune modulating drugs, metabolic substances, corticosteroids to traditional Chinese medicine. Recently, many agents such as tacrolimus, 10-hydroxycamptothecin, and tamoxifen, suberolyanilide hydroxamic acid, apigenin and emodin has been verified to be effective in inhibiting fibroblast proliferation and reduce epidural fibrosis (20-22). Gels including ADCON-L, sodium hyaluronate, Gel Amidon Oxyde, high molecular weight hyaluronan, poly(D,L-lactic acid-co-glycolic acid)- poly(ethylene glycol)-poly(D,L-lactic acid-co-glycolic acid) (PLGA-PEG-PLGA) thermogel were also investigated as promising devices that could reduce epidural scaring and dural adhesion (23). All these agent and gels have shown limited clinical efficacy 
Table 2. For each given hour different CSF percentages were compared to each other. Mean difference of each comparison and its statistical significance is indicated. The degree of significance is indicated with the number of ${ }^{\star}$, higher the number of * stronger the significance.

\begin{tabular}{|c|c|c|c|}
\hline Holm-Sidak's multiple comparisons test & Mean Diff. & Significant? & Summary \\
\hline \multicolumn{4}{|l|}{24 Hours } \\
\hline 0 vs. 25 & -1496 & Yes & $* * *$ \\
\hline 0 vs. 50 & -1238 & Yes & $* * *$ \\
\hline 0 vs. 75 & -516.8 & No & ns \\
\hline 0 vs. 100 & 392 & No & ns \\
\hline 25 vs. 50 & 258.1 & No & ns \\
\hline 25 vs. 75 & 979.5 & Yes & ** \\
\hline 25 vs. 100 & 1888 & Yes & $* * * *$ \\
\hline 50 vs. 75 & 721.4 & Yes & * \\
\hline 50 vs. 100 & 1630 & Yes & $* * * *$ \\
\hline 75 vs. 100 & 908.8 & Yes & $* *$ \\
\hline \multicolumn{4}{|l|}{48 Hours } \\
\hline 0 vs. 25 & -1260 & Yes & $* * *$ \\
\hline 0 vs. 50 & -1338 & Yes & $* * *$ \\
\hline 0 vs. 75 & -496.3 & No & ns \\
\hline 0 vs. 100 & 908.9 & Yes & $* *$ \\
\hline 25 vs. 50 & -78.19 & No & ns \\
\hline 25 vs. 75 & 763.9 & Yes & * \\
\hline 25 vs. 100 & 2169 & Yes & $* * * *$ \\
\hline 50 vs. 75 & 842.1 & Yes & * \\
\hline 50 vs. 100 & 2247 & Yes & $* * * *$ \\
\hline 75 vs. 100 & 1405 & Yes & $* * *$ \\
\hline \multicolumn{4}{|l|}{72 Hours } \\
\hline 0 vs. 25 & -1159 & Yes & $* *$ \\
\hline 0 vs. 50 & -1301 & Yes & $* * *$ \\
\hline 0 vs. 75 & -369.5 & No & ns \\
\hline 0 vs. 100 & 1307 & Yes & $* * *$ \\
\hline 25 vs. 50 & -142.1 & No & ns \\
\hline 25 vs. 75 & 789 & Yes & * \\
\hline 25 vs. 100 & 2466 & Yes & $* * * *$ \\
\hline 50 vs. 75 & 931.1 & Yes & $* *$ \\
\hline 50 vs. 100 & 2608 & Yes & $* * * *$ \\
\hline 75 vs. 100 & 1677 & Yes & $* * * *$ \\
\hline
\end{tabular}

until now. The clinical application of most of these chemicals are limited due to various reasons such as drug toxicity and adverse effects (9). Perhaps what is also very common regarding these chemicals is that all of them come at a certain monetary cost to the patient but the use of CSF, their own, would be of no monetary consequence.

Cerebrospinal fluid, as the name suggests, is a clear and colourless liquid found around the brain and spinal cord. It is derived from the blood and secreted by choroid plexus (CP) in the ventricles of the brain. Ependymal cells of choroid plex- us produce cerebrospinal fluid continuously. The main task of cerebrospinal fluid is to provide mechanical and immunological protection for the central nervous system (24). An average adult is thought to have around $150 \mathrm{ml}$ of CSF, produced at a rate of $0,3-0,4 \mathrm{ml} / \mathrm{min}(430-530 \mathrm{ml} /$ day $)(25,26)$. The CSF is thought to be $99 \%$ water and remaining $1 \%$ is made up of proteins, glucose, neurotransmitters and ions. Trace amounts of cell and immunoglobulins can also be found in the CSF (less than 5 cells $/ \mathrm{ml}$ ). The protein content and concentrations vary with different pathologies and from person to person (25-27). 
Table 3. Comparisons of different time points within different CSF percentages. Mean difference of each comparison and its statistical significance is indicated. The degree of significance is indicated with the number of ${ }^{*}$, higher the number of ${ }^{*}$ stronger the significance.

\begin{tabular}{|c|c|c|c|}
\hline Holm-Sidak's multiple comparisons test & Mean Diff. & Significant? & Summary \\
\hline \multicolumn{4}{|l|}{$0 \%$ CSF } \\
\hline 24 Hours vs. 48 Hours & -1087 & Yes & $* * *$ \\
\hline 24 Hours vs. 72 Hours & -1866 & Yes & $* * * *$ \\
\hline 48 Hours vs. 72 Hours & -778.6 & Yes & $* *$ \\
\hline \multicolumn{4}{|l|}{$25 \% \mathrm{CSF}$} \\
\hline 24 Hours vs. 48 Hours & -850.9 & Yes & ** \\
\hline 24 Hours vs. 72 Hours & -1528 & Yes & $* * * *$ \\
\hline 48 Hours vs. 72 Hours & -677 & Yes & * \\
\hline \multicolumn{4}{|l|}{$50 \%$ CSF } \\
\hline 24 Hours vs. 48 Hours & -1187 & Yes & $* * *$ \\
\hline 24 Hours vs. 72 Hours & -1928 & Yes & $* * * *$ \\
\hline 48 Hours vs. 72 Hours & -740.9 & Yes & ** \\
\hline \multicolumn{4}{|l|}{ 75\% CSF } \\
\hline 24 Hours vs. 48 Hours & -1066 & Yes & $* * *$ \\
\hline 24 Hours vs. 72 Hours & -1718 & Yes & $* * * *$ \\
\hline 48 Hours vs. 72 Hours & -651.9 & Yes & * \\
\hline \multicolumn{4}{|l|}{$100 \%$ CSF } \\
\hline 24 Hours vs. 48 Hours & -570.1 & No & ns \\
\hline 24 Hours vs. 72 Hours & -950.3 & Yes & $* *$ \\
\hline 48 Hours vs. 72 Hours & -380.3 & No & ns \\
\hline
\end{tabular}

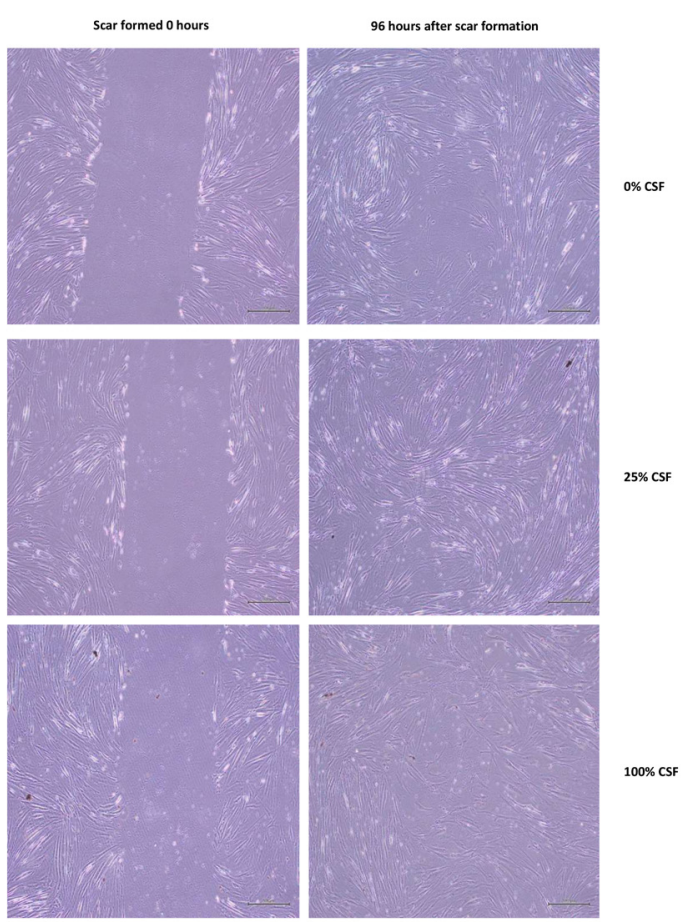

Figure 3. Wound healing assay where an $80 \%$ confluent well was scratched with a pipet tip to create the "scar". Cells are then allowed to close the scar under different CSF percentages. 0 hours and 96 hours after scar formation is shown above for 0\%, 25\% and 100\% CSF. 25\% CSF almost completely closes the wound. $0 \%$ CSF mostly closes the wound but patches of unclosed sections are still apparent. $100 \%$ CSF fails to close the wound. Some cells can be seen reaching into the wound. 
Diverse molecules such as miRNA, hormones and lipids may even be present in the CSF (25).

In our experimental in vitro study, the strongest difference of cell growth was observed when the $25 \%$ and $50 \%$ CSF exposed cells are compared to the $100 \%$ CSF exposed cells in all the measurement times $(24 \mathrm{~h}, 48 \mathrm{~h}$ and $72 \mathrm{~h})$. CSF, in general, contains a higher concentration of sodium, chloride and magnesium and lower concentrations of potassium and calcium when compared to plasma (25). As a result of the lower concentrations of potassium, intracellular potassium in fibroblasts is probably depleted as well. These fibroblasts with the depleted potassium lose their ability for attachment and spreading which are necessary for fibrosis (28). This leads us to hypothesize that the fibroblasts in our study lost their ability to spread and attach probably due to the low potassium concentrations of CSF in the experiment. We do not think that the CSF is having such a toxic effect that the cells are unable to survive at all as the cells were still viable in their flask even after 144 hours of culture (data not shown). The CSF may be able to sustain the cells but through a separate mechanism stop fibroblasts from proliferating.

There are limited amounts of immunoglobulins and negligible amounts of white blood cells in the CSF when compared to plasma and lymph $(27,29)$. After the traumatic injury caused during surgery, acute inflammation occurs through cells such as the PNL, monocytes, macrophages. At some point, fibroblasts enter the scene, eventually leading to end result phenomenon that we call fibrosis. Presence of enough CSF during this inflammation and fibrosis stage, can dilute out the effect and impair fibroblast activity.

CSF has never been studied in any experimental model as possible agent for EF prevention. CSF will have some significant differences and advantages compared to artificial chemicals and chemicals derived from other sources. As it is naturally a body produced material, there will not be an issue of toxicity to the person. Additionally, it will be much cheaper and easier to obtain compared to some of the other chemicals tried. As we will be using the person's own CSF for themselves we do not foresee any issues with immunogenicity of the biomaterial also. As Table 1 indicated the differences observed using different CSF percentages were very significant whereas the time (in hours) was not significant. This could be taken to mean that the different CSF concentrations are showing significant effect in cell proliferation regardless of the time point. This could be seen in Table 2 as well as the percentage comparisons are almost identical in the different time points. It is very difficult to compare this finding to the current literature as this is the first time the CSF is used to look at fibroblast proliferation. CSF has been shown to affect proliferation and migration in stem cells but not in fibroblasts (30). There are examples of either reduced proliferation with different chemicals or no major difference observed different systems used. However, these systems include animal models, non-comparable setup and/or different chemicals that make it difficult for us to compare our results $(8,10,19)$.
Experiments were done using three CSF samples from three different individuals. Each experiment was performed in quadruplicate to account for variability due to pipetting and other experimental errors. The results obtained with CSF1 and 2 were very similar to each other. Although CSF3 presents a similar patter to 1 and 2 the cell numbers were drastically higher. This could be due to individual variability between the different CSFs. As they come from different individuals, there could be subtle differences in protein/hormone, miRNA or ionic content which could result in a higher growth potential for the cells. This is one of the possible limitations of our study. Perhaps one potential way to pursue this in the future would be to analyse each CSF for content in detail. When the literature was scanned there were examples of pooling the CSF $(31,32)$. However, we do believe that there is a value to using separate CSFs and catching subtle differences. What way be seen as a limitation could also allow us to do a separate analysis which we would not have been able to do otherwise. We still need to point out that we may not have been able to figure out the growth difference as it may be due to multiple factors all at once.

There were some limitations to the study. To begin with we were only able to do the experiment with CSF from 3 different people. A higher number will definitely lead to more statistically valuable results, but it is important to remember that CSF is difficult to obtain in large amounts. And even with 3 individuals we were able to get statistically significant results. Another way around this problem would be to pool smaller CSF samples from different people to get enough CSF for a complete experiment. However, with that option we may be losing our ability to observe individual variation, which we wanted to see. As mentioned earlier, interpersonal CSF differences and our inability to determine where the variation is coming from is another limitation of our study and we plan to do a full CSF content analysis in future experiments. Another limitation of our study is the use of skin fibroblast cells only. There could be other fibroblast contributions to fibrosis in EP such as the muscle fibroblasts. Testing fibroblasts from different sources to see their response to CSF is a future goal.

It is also important to keep in mind that in vitro experiments are no substitute for the actual conditions in in vivo environments, but they do provide a controlled starting point to investigate new paths.

The molecular mechanism though which the CSF is exerting its effect on the fibroblasts will also need to be determined. This may be achieved through looking at the expression profiles of genes in the apoptotic and proliferative pathways. Another possible molecular analysis could be done through global expression profiling of genes and miRNA in fibroblasts with exposure to varying percentages of CSF. A recent study by Wu. Et al has looked at the effect of mocetionstat and has observed that it exerts its effects through HDAC1 on the AKT/GSK2b pathway to suppress epidural fibrosis (33). A similar molecular profiling for CSF remains a future goal. Another future goal for is to combine CSF with materials such as PLGA that may regulate the release of the CSF over time and lead to a better effect. 


\section{Conclusion}

In conclusion, adverse effects of low concentrations of potassium levels and dilutional effect of CSF may be a promising solution in the prevention of EF. The exact application method and duration of the application will need to be determined following further in vitro and in vivo experiments, which will include animal experiments (with rats).

\section{Funding}

This research was funded in full by the Near East University, Research Center of Experimental Health Sciences (DESAM), Nicosia, Cyprus.

\section{Conflicts of interest/Competing interests:}

The authors declare no conflicts of interest.

\section{Ethics approval}

University Scientific Research Ethics Evaluation Board (YDU/2017/44-372)

\section{Acknowledgements}

We would like to thank Assist. Prof. Dr. Özgür Tosun for his help and guidance.

\section{References}

1. Chen H, Yan L, Wang J, Sun Y, Li X, Zhao S, et al. Methotrexate prevents epidural fibrosis through endoplasmic reticulum stress signalling pathway. Eur J Pharmacol. 2017;796(December 2016):131-8.

2. Guyer RD, Patterson M, Ohnmeiss DD. Failed back surgery syndrome: Diagnostic evaluation. Vol. 14, Journal of the American Academy of Orthopaedic Surgeons. American Association of Orthopaedic Surgeons; 2006. p. 534-43.

3. Emmez H, Kardes O, Dogulu F, Kurt G, Memis L, Baykaner MK. Role of antifibrotic cytokine interferon- $\gamma$ in the prevention of postlaminectomy peridural fibrosis in rats. Neurosurgery. 2008 Jun;62(6):1351-7.

4. Lv P, Zhao J, Su W, Liang X, Zhang K. An experimental novel study: hyperbaric oxygen treatment on reduction of epidural fibrosis via down-regulation of collagen deposition, IL-6, and TGF- $\beta 1$. Eur J Orthop Surg Traumatol (Internet). 2015;25:53-8. Available from: http://dx.doi. org/10.1007/s00590-014-1509-x

5. Turkoglu E, Dinc C, Tuncer C, Oktay M, Serbes G, Sekerci Z. Use of decorin to prevent epidural fibrosis in a post-laminectomy rat model. Eur J Pharmacol (Internet). 2014 Feb 5 (cited 2021 Jan 20);724(1):86-91. Available from: https:// pubmed.ncbi.nlm.nih.gov/24374200/

6. Gürer B, Kahveci R, Gökçe EC, Ozevren H, Turkoglu E, Gökçe A. Evaluation of topical application and systemic administration of rosuvastatin in preventing epidural fibrosis in rats. Spine J. 2015;15(3):522-9.

7. Zhang C, Kong X, Liu C, Liang Z, Zhao H, Tong W, et al. ERK2 small interfering RNAs prevent epidural fibrosis via the efficient inhibition of collagen expression and inflam- mation in laminectomy rats. Biochem Biophys Res Commun (Internet). 2014;444(3):395-400. Available from: http://dx.doi.org/10.1016/j.bbrc.2014.01.070

8. Liu ZC, Li Y, Zang Y, Cui G, Sang HX, Ma ZS, et al. Clinical assessment of a CMC/PEO gel to inhibit postoperative epidural adhesion formation after lumbar discectomy: A randomized, control study(1) Z.C. Liu, Y. Li, Y. Zang, G. Cui, H.X. Sang, Z.S. Ma, et al., Clinical assessment of a CMC/PEO gel to inhibit. Arch Orthop Trauma Surg. 2013;133(3):295-301.

9. Zhang K, Zhao J, Su W, Lu R, Lv P. Immunomodulatory effectiveness of licofelone in preventing epidural fibrosis in post-laminectomy rat. Eur J Orthop Surg Traumatol (Internet). 2015;25:63-8. Available from: http://dx.doi. org/10.1007/s00590-014-1534-9

10. Sun Y, Ge Y, Fu Y, Yan L, Cai J, Shi K, et al. Mitomycin C induces fibroblasts apoptosis and reduces epidural fibrosis by regulating MIR-200b and its targeting of RhoE. Eur J Pharmacol (Internet). 2015;765:198-208. Available from: http://dx.doi.org/10.1016/j.ejphar.2015.08.002

11. Ramirez LF, Thisted R, Sypert GW, Horwitz N. Complications and demographic characteristics of patients undergoing lumbar discectomy in community hospitals. Neurosurgery. 1989;25(2):226-31.

12. Goodkin R, Laska LL. Unintended "incidental” durotomy during surgery of the lumbar spine: medicolegal implications. Surg Neurol (Internet). 1995 Jan (cited 2019 Nov 18);43(1):4-12; discussion 12-4. Available from: http:// www.ncbi.nlm.nih.gov/pubmed/7701421

13. Greenberg MS, Greenberg MS. Handbook of neurosurgery. Greenberg Graphics; 2010. 1337 p.

14. Zhang C, Kong X, Ning G, Liang Z, Qu T, Chen F, et al. Alltrans retinoic acid prevents epidural fibrosis through NF$\kappa \mathrm{B}$ signaling pathway in post-laminectomy rats. Neuropharmacology (Internet). 2014;79:275-81. Available from: http://dx.doi.org/10.1016/j.neuropharm.2013.11.010

15. Wang J, Dodd C, Shankowsky HA, Scott PG, Tredget EE. Deep dermal fibroblasts contribute to hypertrophic scarring. Lab Investig (Internet). 2008 Dec (cited 2021 Jan 20);88(12):1278-90. Available from: https://pubmed.ncbi. nlm.nih.gov/18955978/

16. Thannickal VJ, Toews GB, White ES, Lynch JP, Martinez FJ. Mechanisms of pulmonary fibrosis (Internet). Vol. 55, Annual Review of Medicine. Annu Rev Med; 2004 (cited 2021 Jan 20). p. 395-417. Available from: https://pubmed. ncbi.nlm.nih.gov/14746528/

17. Ozturk Y, Bozkurt I, Yaman ME, Guvenc Y, Tolunay T, Bayram P, et al. Histopathologic Analysis of Tamoxifen on Epidural Fibrosis. World Neurosurg. 2018 Mar 1;111:e941-8.

18. Farrokhi MR, Vasei M, Fareghbal S, Farrokhi N. The effect of methylene blue on peridural fibrosis formation after laminectomy in rats: An experimental novel study. Spine J (Internet). 2011;11(2):147-52. Available from: http://dx.doi.org/10.1016/j.spinee.2011.01.014 
19. Bolat E, Kocamaz E, Kulahcilar Z, Yilmaz A, Topcu A, Ozdemir M, et al. Investigation of efficacy of mitomycin-C, Sodium hyaluronate and human amniotic fluid in preventing epidural fibrosis and adhesion using a rat laminectomy model. Asian Spine J. 2013 Dec;7(4):253-9.

20. Jiao R, Chen H, Wan Q, Zhang X, Dai J, Li X, et al. Apigenin inhibits fibroblast proliferation and reduces epidural fibrosis by regulating Wnt3a / $\beta$-catenin signaling pathway. 2019;8:1-9.

21. Yang J, Liu P, Liu J, Chu L, Li J, Chen C, et al. Suberoylanilide hydroxamic acid attenuates epidural fibrosis via inhibiting myofibroblast differentiation and increasing fibroblast apoptosis. 2020;5726-33.

22. Xiong G, Chen H, Wan Q, Dai J, Sun Y, Wang J, et al. Emodin promotes fibroblast apoptosis and prevents epidural fibrosis through PERK pathway in rats. 2019;9:1-7.

23. D PEG, Li X, Chen L, Lin H, Cao L, Cheng J, et al. Efficacy of Poly ( D , L -Lactic Acid-co-Glycolic acid ) - Acid ) Thermogel As a Barrier to Prevent Spinal Epidural Fibrosis in a Postlaminectomy Rat Model. 2017;30(3):283-90.

24. Williams PL. Gray's anatomy: the anatomical basis of clinical practice. 38th Ed. Bannister LH, editor. Churchill Livingstone Elsevier; 1995. 1202 p.

25. Khasawneh A, Garling R, Harris C. Cerebrospinal fluid circulation: What do we know and how do we know it? Brain Circ. 2018;4(1):14.

26. Telano LN, Baker S. Physiology, Cerebral Spinal Fluid (CSF) (Internet). StatPearls. 2018 (cited 2019 Nov 25). Available from: http://www.ncbi.nlm.nih.gov/pubmed/30085549

27. Spector R, Robert Snodgrass S, Johanson CE. A balanced view of the cerebrospinal fluid composition and functions: Focus on adult humans. Vol. 273, Experimental Neurology. Academic Press Inc.; 2015. p. 57-68.

28. Altankov G, Grinnell F. Depletion of intracellular potassium disrupts coated pits and reversibly inhibits cell polarization during fibroblast spreading. J Cell Biol (Internet). 1993 (cited 2021 Jan 20);120(6):1449-59. Available from: https://pubmed.ncbi.nlm.nih.gov/8449988/

29. Wake H, Moorhouse AJ, Jinno S, Kohsaka S, Nabekura J. Resting microglia directly monitor the functional state of synapses in vivo and determine the fate of ischemic terminals. J Neurosci (Internet). 2009 Apr 1 (cited 2021 Jan 20);29(13):3974-80. Available from: https://pubmed.ncbi. nlm.nih.gov/19339593/

30. Zhu M, Feng Y, Dangelmajer S, Guerrero-Cázares H, Chaichana KL, Smith CL, et al. Human cerebrospinal fluid regulates proliferation and migration of stem cells through insulin-like growth factor-1. Stem Cells Dev. 2015 Jan 15;24(2):160-71.

31. Bjorefeldt A, Andreasson U, Daborg J, Riebe I, Wasling P, Zetterberg $\mathrm{H}$, et al. Human cerebrospinal fluid increases the excitability of pyramidal neurons in the in vitro brain slice. J Physiol. 2015 Jan 1;593(1):231-43.

32. Matzneller P, Burian A, Zeitlinger M, Sauermann R. Understanding the Activity of Antibiotics in Cerebrospinal
Fluid in vitro. Pharmacology (Internet). 2016 Feb 17 (cited 2019 Nov 26);97(5-6):233-44. Available from: https:// www.karger.com/Article/FullText/444263

33. Wu WJ, Wang J, Liang J, Zhou Q, Liang Y. Mocetinostat suppresses epidural fibrosis following laminectomy by inhibiting myofibroblast activation and increasing apoptosis. Eur Rev Med Pharmacol Sci (Internet). 2020 (cited 2021 Mar 30);24(8):4467-75. Available from: https://pubmed. ncbi.nlm.nih.gov/32373984/ 